\title{
Food preference for milk and dairy products
}

\author{
Zuzana Derflerová Brázdová1, Helena Klimusová ${ }^{2}$, Lenka Vorlová3 Jindřich Fiala $^{1}$
${ }^{1}$ Masaryk University, Faculty of Medicine, Department of Preventive Medicine, ${ }^{2}$ Faculty of Arts, Department of Psychology, Brno, Czech Republic Department of Milk Hygiene and Technology, Brno, Czech Republic \\ ${ }^{3}$ University of Veterinary and Pharmaceutical Sciences, Faculty of Veterinary Hygiene and Ecology,
}

Received March 21, 2014

Accepted September 17, 2014

\begin{abstract}
Milk and dairy products constitute an important source of energy and nutrients for humans. Food preferences may significantly influence the actual consumption (and thus nutrition) of people at the population level. The objective of the present large-scale survey was to specify current preferences for milk and dairy products with regard to age and sex. The study was conducted across the Moravia region, Czech Republic, on a sample of 451 individuals divided into 4 age groups: children, adolescents, young adults, and elderly people. A graphic scale questionnaire was administered, with respondents rating their degree of preference for each food item by drawing a mark on a $35 \mathrm{~mm}$ line. Out of the 115 items in the questionnaire, 11 items represented dairy products. Data was analysed by means of a general linear model using IBM SPSS Statistics software. Preference for milk was lower in the elderly group than the other groups $(P<0.01)$. In children, the most preferred dairy product was cream, which was in contrast with the elderly group. Significant sex differences were only found with low-fat yoghurt $(P<0.01)$. The overall preference for dairy products (21.6) was lower than the average preference for all foods on the list (22.5). The cross-sectional study revealed intergenerational differences in preferences for specific dairy products, which were most marked in case of cream, processed cheese, blue cheese, and buttermilk. The knowledge of these differences might help promote more focused action at the community level directed at increasing the overall consumption of dairy products in the population.
\end{abstract}

Food group, food liking, intergenerational differences, gender differences

Food liking or food preferences in humans are formed on the basis of interaction between innate traits, e.g. preference for sweet taste in mammals (Breen et al. 2006) and cognitively acquired attitudes to various kinds of food, i.e. repeated experience (Harris 2008). Breast milk is the only food which is truly 'natural' to the human individual up to the sixth month of age. All other foods in the human diet are a result of artificial selection. The fact that milk of other mammalian species (cow milk, etc.) in our culture represents a certain follow-up of breast milk consumption makes - especially in childhood - intake of milk easier in comparison with intake of foods with other organoleptic properties (Ramos and Stein 2000; Janstova et al. 2011). Still, general preference for milk decreases later in life. In some populations, higher prevalence of lactose intolerance might contribute to the development of milk aversion. However, in Europe, the prevalence of lactose intolerance (which does not exceed 25\%) is lower than in any other continent (Bhatnagar and Aggarwall 2007).

\section{Materials and Methods}

The GraphScale35 Questionnaire, developed by authors, for the assessment of food liking/food preference was used with 115 food items. During the development of the questionnaire, several versions were piloted by authors in the years 1997-2012, originally with 148 food items; the final version of the questionnaire has a high degree of internal consistency and clear factor structure. Milk and dairy products together constituted $9 \%$ of all items. The respondents were asked to mark their degree of preference for each food graphically on a $35 \mathrm{~mm}$ line. The score for each item was coded as a whole number ranging from 0 (least preferred) to 35 (most preferred). 
The research sample consisted of 451 respondents residing in the Moravia Region, Czech Republic. The method of selection was a combination of probability and non-probability sampling. The proportion of age subgroups was predetermined by stratified sampling method; respondents within age subgroups therefore were recruited by convenience sampling. The sample was divided into four age groups representing specific life periods: children, adolescents, young adults, and elderly people. The proportion of females ranged from $45 \%$ in the children's group to $78 \%$ in the elderly group. Descriptive statistics of the sample are summarized in Table 1 . A majority of respondents $(85.6 \%)$ came from urban regions (city or town).

Statistical analysis of the obtained data was performed using the IBM SPSS Statistics software. The effects of sex and age on food preferences were tested by means of a general linear model (GLM). Differences were considered significant at $P<0.05$.

Table 1. Descriptive statistics of the total sample and the four age group subsamples.

\begin{tabular}{|c|c|c|c|c|c|c|}
\hline Age group & $\begin{array}{c}\mathrm{N} \\
(\mathrm{F} / \mathrm{M})\end{array}$ & $\begin{array}{c}\text { Sex } \\
\text { Mean }\end{array}$ & $\begin{array}{l}\text { Age } \\
\text { SD }\end{array}$ & Median & Range & \\
\hline Children & 115 & $52 / 63$ & 11.9 & 0.81 & 12 & $11-13$ \\
\hline Adolescents & 135 & $71 / 64$ & 15.4 & 0.67 & 16 & $14-16$ \\
\hline Young adults & 102 & $71 / 31$ & 27.7 & 0.96 & 24 & $23-28$ \\
\hline Elderly & 99 & $77 / 22$ & 68.1 & 4.90 & 67 & $60-82$ \\
\hline Total & 451 & $280 / 191$ & 28.9 & 21.82 & 16 & $11-82$ \\
\hline
\end{tabular}

$\mathrm{N}$ - number; F/M - females/males; SD - standard deviation

\section{Results}

Using the scale 0 to 35 , the average score of food liking for all 11 dairy products on the food list was 21.6. The average preference for all 42 foods of animal origin, including dairy

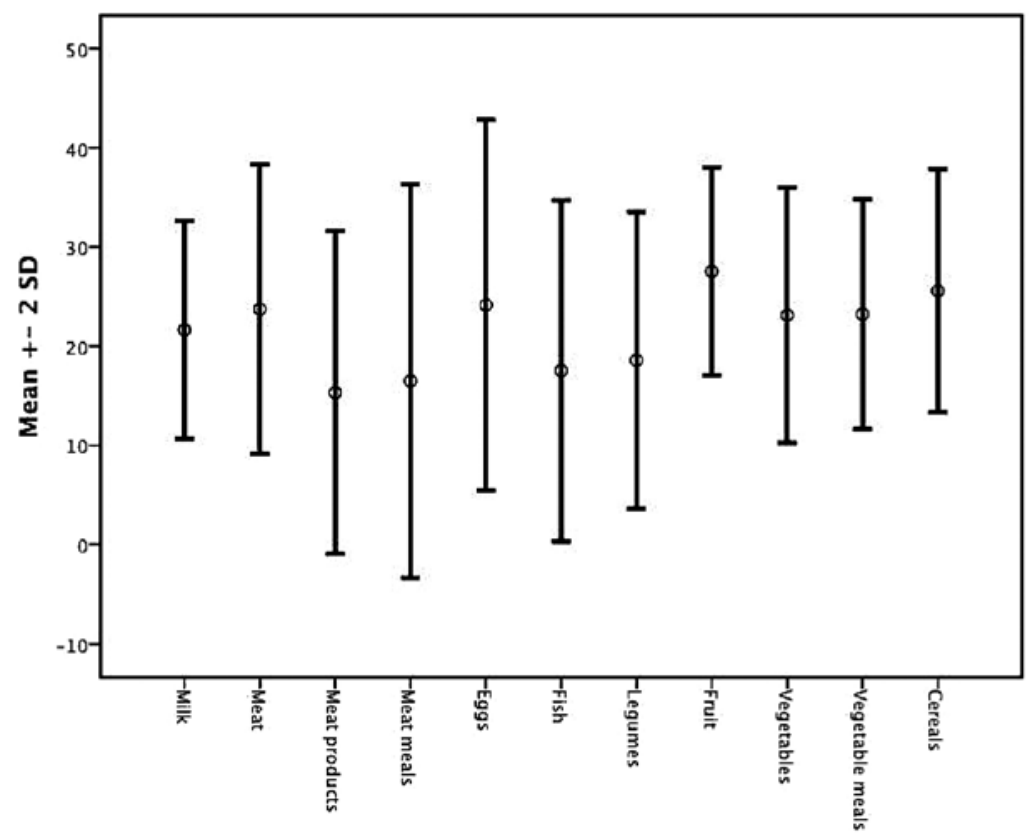

Fig. 1. Means plot of average liking for all food categories 
products, was 19.1, which was less than the overall average of 22.5 encompassing all 115 food items in the questionnaire.

When the list of 115 foods was divided into 11 categories, the group incorporating dairy products ranked among moderately preferred categories (Fig. 1). The three most favoured categories included fruits, followed by cereals and eggs. Food groups with medium average preference scores included meat, vegetables, vegetable meals, and dairy products. Low variance in preference scores in dairy products indicated that respondents' ratings of this category tended to converge (the same was true for fruits). Greater variance was observed in less preferred foods, such as fish, meat products, meat meals, and legumes.

Within the category of dairy products the most preferred commodities were hard cheese, milk, cream, butter, and creamy yoghurt. The least preferred dairy products, on the other hand, were buttermilk, processed cheese, and low-fat yoghurt. We used GLM to test sex differences and differences between the four age groups. Whereas the main effect of age was significant for all dairy foods except creamy yoghurt, a significant main effect of sex was only found in a single item, i.e. low-fat yoghurt $(\mathrm{F}=10.8, P<0.01)$, which was more preferred by women $(\mathrm{M}=19.6)$ than men $(\mathrm{M}=16.9)$. No interaction effects were observed throughout the food group. Considering age differences more specifically, preference for milk was substantially lower in the elderly group than that in any other group. The reverse was true for buttermilk, which was significantly more preferred in the elderly group. Liking for processed cheese seems to decrease with age, while liking for hard cheese, blue cheese and kefir slightly increases. Curd cheese is more likely to be preferred by adolescents and young adults than children or the elderly. In the children's group, a special position was occupied by cream, which was the most preferred dairy product with a median of 35 (i.e. more than one half of the children marked a maximum degree of preference for this food). In older groups preference for cream was visibly lower; the elderly group even rated it as one of less preferred dairy products.

\section{Discussion}

Food preference for milk and dairy products is strongly influenced by historical and cultural factors. Countries with pastoral tradition do not only show higher overall preference for dairy products at the population level, but also a higher degree of actual milk consumption (Finland, the Netherlands, etc.). Attitudes of various age groups towards milk and dairy products might be both positively and negatively shaped by food advertising in media, as well as by one's adherence to specific lifestyles (Byrnes and Hayes 2013) incorporating various taboos and to cultural symbolism which makes milk consumption problematic or completely undesirable, e.g. veganism (Crockett and Sims 1995; Story et al. 2002). Our study is limited by its cross-sectional, non-longitudinal design. Nevertheless, the intergenerational differences in preference for milk, cream, butter, hard cheese, flavoured curd cheese, etc., can be to a large degree attributed to the natural physiological changes occurring in the human organism throughout the lifespan (Ton $\mathrm{Nu}$ et al. 1996; Nicklaus et al. 2004; Cooke and Wardle 2005). Similarly, the sex difference in liking for low-fat yoghurt probably has a complex basis, associated with the observation that women are generally more likely to engage in positive health behaviours than men are (Fiala and Brazdova 2000; Diehl 2009). A substantially lower degree of preference for cream in the older groups apparently demonstrates a general decrease in preference for fatty foods later in life, which can be affected by higher occurrence of gastrointestinal disorders in the old age. Butter might be an exception to this pattern perhaps because it is generally consumed in relatively low amounts. An example of a highly preferred food type in young adults is blue cheese, which together with olives, fish or seafood illustrates a general preference for savoury and strong flavours in this age group. At the same time, young adults are more 
likely to favour 'healthy' foods (Drewnowski and Hahn 1999), which is reflected in increased preference for kefir and cottage cheese, and decreased preference for processed cheese. The awareness of age differences in preferences for specific food types might provide a basis for more focused action at the community level directed at increasing the overall consumption of milk and dairy products in the population (Eertmans et al. 2001).

\section{References}

Bhatnagar S, Aggarwal R 2007: Lactose intolerance. BMJ 334: 1331-1332

Breen F, Plomin R, Wardle J 2006: Heritability of food preferences in young children. Physiol Behav 88: $443-447$ Byrnes NK, Hayes JE 2013: Personality factors predict spicy food liking and intake. Food Qual Prefer 28: 213221

Cooke LJ, Wardle J 2005: Age and gender differences in children's food preferences. Br J Nutr 93: 741-746

Crockett SJ, Sims LS 1995: Environmental influences on children's eating. J Nutr Educ 27: 235-250

Diehl JM 2009: Food preferences of 10- to 14-year-old boys and girls. Schweiz Med Wochenschr 129: 151-161

Drewnowski A, Hahn C 1999: Food preferences and reported frequencies of food consumption as predictors of current diet in young women. Am J Clin Nutr 70: 28-36

Eertmans A, Baeyens F, Van den Bergh O 2001: Food likes and their relative importance in human eating behavior: review and preliminary suggestions for health promotion. Health Educ Res 16: 443-456

Fiala J, Brazdova Z 2000: A comparison between the lifestyles of men and women - parents of school age children. Cent Eur J Public Health 8: 94-100

Harris G 2008: Development of taste and food preferences in children. Curr Opin Clin Nutr Metab Care 11: 315-319

Janstova B, Drackova M, Dleskova K, Cupakova S, Necidova L, Navratilova P, Vorlova L 2011: Quality of raw milk from a farm with automatic milking system in the Czech Republic. Acta Vet Brno 80: 207-214

Nicklaus S, Boggio V, Chabanet C, Issanchou S 2004: A prospective study of food preferences in childhood. Food Qual Prefer 15: 805-818

Ramos M, Stein LM 2000: Development children's eating behavior. J Pediatr (Rio J) 76: S229-S237

Story M, Neumark-Sztainer D, French S 2002: Individual and environmental influences on adolescent eating behaviors. J Am Diet Assoc 102: S40-S51

Ton Nu Ch, MacLeod P, Barthelemy J 1996: Effects of age and gender on adolescents' food habits and preferences. Food Qual Prefer 7: 251-262 